\title{
Investigating and Classifying the Applications of Flexible Manufacturing Systems in Customization
}

\author{
${ }^{1}$ Ahmad Afsari \\ ${ }^{I}$ Department of Mechanical Engineering, Faculty of Engineering, Islamic Azad University Shiraz Branch, Fars, \\ Iran
}

\begin{abstract}
The recent manufacturing environment is characterized as having diverse products due to mass customization, short production lead-time, and unstable customer demand. Today, the need for flexibility, quick responsiveness, and robustness to system uncertainties in production scheduling decisions has increased significantly. In traditional job shops, tooling is usually assumed as a fixed resource. However, when tooling resource is shared among different machines, a greater product variety, routing flexibility with a smaller tool inventory can be realized. Such a strategy is usually enabled by an automatic tool changing mechanism and tool delivery system to reduce the time for tooling setup, hence allows parts to be processed in small batches. In this research, a dynamic scheduling problem under flexible tooling resource constraints is studied. An integrated approach is proposed to allow two levels of hierarchical, dynamic decision making for job scheduling and tool flow control in Automated Manufacturing Systems. It decomposes the overall problem into a series of static subproblems for each scheduling window, handles random disruptions by updating job ready time, completion time, and machine status on a rolling horizon basis, and considers the machine availability explicitly in generating schedules. Two types of manufacturing system models are used in simulation studies to test the effectiveness of the proposed dynamic scheduling approach. First, hypothetical models are generated using some generic shop flow structures (e.g. flexible flow shops, job shops, and single-stage systems) and configurations(Insup,Um.,et al.,2009). They are tested to provide the empirical evidence about how well the proposed approach performs for the general automated manufacturing systems where parts have alternative routings. Second, a model based on a real industrial flexible manufacturing system was used to test the effectiveness of the proposed approach when machine types, part routing, tooling, and other production parameters closely mimic to the real flexible manufacturing operations.
\end{abstract}

Keywords: Flexible manufacturing system; Customization; Agile manufacturing; Distributed control; Automated production

\section{Introduction}

A flexible manufacturing system (FMS) is a manufacturing system in which there is some amount of flexibility that allows the system to react in the case of changes, whether predicted or unpredicted. This flexibility is generally considered to fall into two categories, which both contain numerous subcategories(Insup,Um.,et al.,2009; Mehrabi,M.,2005).

The first category, machine flexibility, covers the system's ability to be changed to produce new product types, and ability to change the order of operations executed on a part. The second category is called routing flexibility, which consists of the ability to use multiple machines to perform the same operation on a part, as well as the system's ability to absorb large-scale changes, such as in volume, capacity, or capability.

Most FMS systems consist of three main systems. The work machines which are often automated CNC machines are connected by a material handling system to optimize parts flow and the central control computer which controls material movements and machine flow.

The main advantage of an FMS is its high flexibility in managing manufacturing resources like time and effort in order to manufacture a new product. The best application of an FMS is found in the production of small sets of products like those from a mass production (Mehrabi,M.,2005; Wilhelm,W.,1986).

To put it in nutshell, the main advantages of FMS are:

Reduced manufacturing times,

Lower cost per unit produced,

Greater labor productivity,

Greater machine efficiency,

Improved quality,

Increased system reliability,

Reduced parts inventories,

Adaptability to CAD/CAM operations,

Shorter lead times. 
To mention, there are two critical disadvantages regarding the application of FMS:

\section{Cost to implement,}

\section{Substantial pre-planning required.}

An Industrial Flexible Manufacturing System (FMS) consists of robots, Computer-controlled Machines, Numerical controlled machines (CNC), instrumentation devices, computers, sensors, and other stand alone systems such as inspection machines. The use of robots in the production segment of manufacturing industries promises a variety of benefits ranging from high utilization to high volume of productivity. Each Robotic cell or node will be located along a material handling system such as a conveyor or automatic guided vehicle. The production of each part or work-piece will require a different combination of manufacturing nodes.

The movement of parts from one node to another is done through the material handling system. At the end of part processing, the finished parts will be routed to an automatic inspection node, and subsequently unloaded from the Flexible Manufacturing System.

The FMS data traffic consists of large files and short messages, and mostly come from nodes, devices and instruments. The message size ranges between a few bytes to several hundreds of bytes. Executive software and other data, for example, are files with a large size, while messages for machining data, instrument to instrument communications, status monitoring, and data reporting are transmitted in small size(Wilhelm,W.,1986; Hung Chen,Jian.,2005).

There is also some variation on response time. Large program files from a main computer usually take about 60 seconds to be down loaded into each instrument or node at the beginning of FMS operation. Messages for instrument data need to be sent in a periodic time with deterministic time delay. Other type of messages used for emergency reporting is quite short in size and must be transmitted and received with almost instantaneous response.

The demands for reliable FMS protocol that support all the FMS data characteristics are now urgent. The existing IEEE standard protocols do not fully satisfy the real time communication requirements in this environment. The delay of CSMA/CD is unbounded as the number of nodes increases due to the message collisions. Token Bus has a deterministic message delay, but it does not support prioritized access scheme which is needed in FMS communications. Token Ring provides prioritized access and has a low message delay, however, its data transmission is unreliable. A single node failure which may occur quite often in FMS causes transmission errors of passing message in that node. In addition, the topology of Token Ring results in high wiring installation and cost.

A design of FMS communication protocol that supports a real time communication with bounded message delay and reacts promptly to any emergency signal is needed. Because of machine failure and malfunction due to heat, dust, and electromagnetic interference is common, a prioritized mechanism and immediate transmission of emergency messages are needed so that a suitable recovery procedure can be applied.

A modification of standard Token Bus to implement a prioritized access scheme was proposed to allow transmission of short and periodic messages with a low delay compared to the one for long messages.

\section{Literature survey}

A considerable body of research literature has accumulated in this area since the late 1970's when the first papers were published. A few surveys of the literature have also appeared (Buzacott and Yao 1986, Rachamadugu and Stecke 1989, Gupta et al. 1989). However, these reviews focused on specific perspectives such as analytical models, or scheduling problems. In this paper we have attempted to review articles having wider methodological perspectives while concentrating on the operations issues. We have also brought the review more up-to-date. We review the literature from multiple viewpoints:

1. Methodology used in resolving the problem

2. Applications viewpoint

3. Time horizon considered

4. FMS factors considered

The concept of flexibility in traditional FMS, which is illustrated in Figure 1, has four major components: volume flexibility, manufacturing flexibility, mix ratio flexibility, and delivery flexibility (Koste/Malhotra, 1998). The MCM system demands a higher degree of flexibility than traditional FMS. It is highly desirable that each component demonstrates prompt response capability in managing demand changes in a FMS with parallel considerations in product costs, quality and reliability to form the flexibility in an agile MCM system, as shown in Figure 1. 


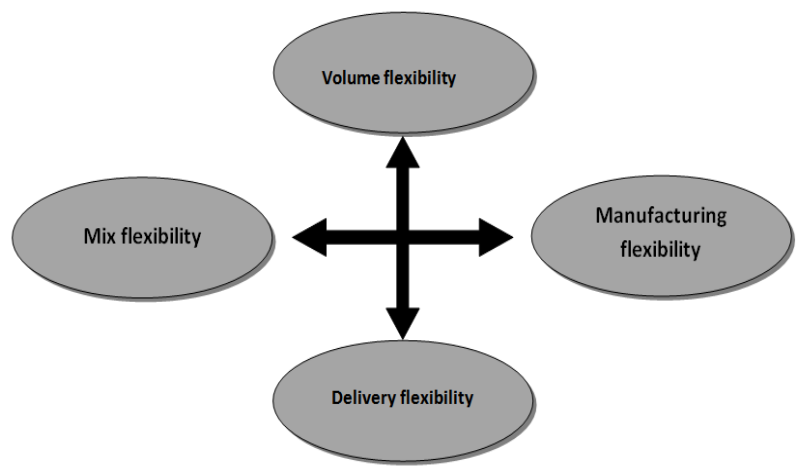

The concept of mass customization was first expounded formally in the book "Future Perfect" by Stanley M. Davis in 1987. In 1993, Joseph Pine (Pine, 1993) gave MCM a clear definition as a strategy that sought to exploit the need to support greater product variety and individualization. Further, the goal of MCM was to produce and deliver customized products rapidly while keeping costs at the mass-production level. Since 1993, advancements to this innovative trend of manufacturing strategy have been drawn from many related knowledge and technology domains (Piller/Stotko, 2002; Kotha, 1996; Tu/Vonderembse/Nathan, 2001; Tait, 2001).

In recent years, advances in computer aided design (CAD), product data management (PDM), and networking technologies have made mass customization no longer a legend, but closer than ever (Ruddy, 2002; Heikkila, 2002). Richard Morley, inventor of the programmable logic controller and co-author of The Technology Machine: How Manufacturing Will Work in the Year 2020, forecasted that, "the word 'personal' will take on more applications: personal families, personal food designed to maximize custom diet needs, personal clothing [clothing sized to individual bodies and fabricated to personal climate and skin needs], and personal [customer-designed] cars" (Felton, 2001). Mass customization is about to take center stage. MCM competent manufacturers will enjoy superior market share and greater profit margins, and it is the promise of these economic incentives that will compel other manufacturers to move to MCM sooner than later.

This strategy brings radical changes to methods used to operate traditional manufacturing enterprises. It is changing the way customers make purchases and has a strong impact on how products are made (Smirnov, 1999). Much of the emerging literature has focused on highlighting the differences between mass-production and mass-customization (Silveira/Borenstein/Fogliatto, 2001). This paper proposes enabling technologies for mass-customization manufacturing systems, and an eXtensible Markup Language (XML) based information integration platform to support MCM.

With the emergence of computers and the introduction of more advanced equipment in industry like Computer Numerically Controlled (CNC) machines, robot manipulators, and automated guided vehicles (AGV), these manufacturing cells gained their share of automation and were hence referred to as Flexible Manufacturing Cells (FMC). Consequently, the production paradigm featuring a group of FMCs supported by an intercellular material handling system was defined as a Flexible Manufacturing System (FMS). These systems have an inherent higher flexibility and functionality than their DMS counterparts and higher output capacity (quantities of products) than job shops as a result of applying GT and automation. These systems hence provide an acceptable balance between DMSs and job shops.

FMSs in industry usually feature a flow shop pattern. That is to say, members of a part family have a restricted uni-flow direction within a cell. With the high levels of automation that these cells feature nowadays, it has been argued that these systems include more functions than what is actually needed. An example would be the incorporation of a highly flexible robot to deliver parts to cater to unidirectional flow between machines rather than a simple conveyor that can accomplish the same task. However, the correct argument should be on how to utilize highly flexible equipment to its functional extent to attain the sought variety, or better yet flexibility, of FMSs. The answer to this argument is the adoption of job shop flow patterns in FMSs. This will not only ensure the full functional utilization of the equipment, but also the flexibility of introducing any product to the system that does not necessarily require the same sequence of operations required by other members of a product family; hence higher functionality (Figure 2). 


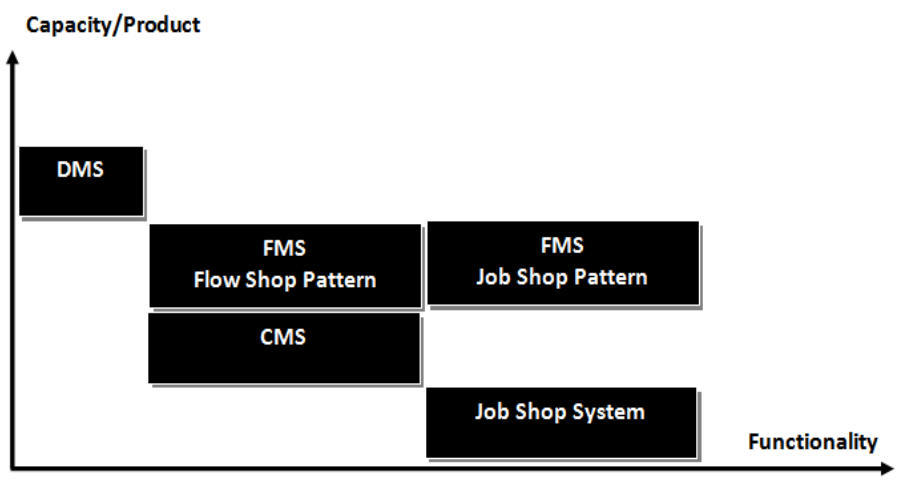

III. Problem definition

Ideally, the functions of a production control system can be classified into three distinct functional modules; a scheduler, a monitor, and a dispatcher. Accordingly, the current study proposes a hierarchical control system divided into an upper level scheduler, and a lower level supervisor that monitors and dispatches commands to the shop floor (Figure 3). The scheduler is responsible for determining a feasible allocation of the resources that optimizes some performance measure, based on the current production requirements and any unforeseen internal or external disruptions. The monitor collects and summarizes shop floor status information and feeds it back to the dispatcher and the scheduler. The dispatcher is then used to sequence and synchronize the physical activities in the system, based on the decisions of the scheduler and the feedback from the monitor.

Realizing the control system shown in Figure 3 can guarantee, not only the correct and safe operation of the controlled system, but also an optimized production performance as follows:

- The scheduler is the decision maker in the control system. According to the current product mix, it provides a production schedule that allocates processing slots for the jobs on the available machines while optimizing some production objective criterion. Taking into consideration the capacities of the available resources, this schedule will further ensure that the resulting job flow cannot cause any deadlock situations(Qiang,Tu.,2001). Upon the occurrence of any internal or external disruption to the system, the scheduler will react to the disruption such that the updated schedule still retains the optimized performance with minimal variations from the original schedule.

- The supervisor is the command executer and observer of the system. In order to implement the original or the updated schedule on the shop floor, the assigned processing slots, and hence the underlying flow plan is transformed into a supervisory format that can interact with the shop floor devices. The supervisor will guarantee that the flow plan (behavior) determined by the scheduler is realized on the shop floor. It evolves in a discrete event manner, and is capable of receiving feedback signals and accordingly issuing action commands directly from/to the shop floor.

To attain and validate this hierarchical control design, the approach followed in this research can be detailed as follows(Zhejun, Gong.,2008):

1. Development of mathematical models for the deadlock-free scheduling problem of flexible job shops that can be solved to obtain optimal schedules while considering a variety of system parameters. The models can then be utilized to schedule a new product mix for small systems, or in the design stages of medium ones.

2. Development of a heuristic, capable of solving the same scheduling problem for larger systems, which cannot be solved optimally using the mathematical formulations due to computational time limitations.

3. Development of a generic tool that can modify the production schedule in a deadlock-free manner to account for common internal or external disruptions in real time, while preserving the production performance and stability of the system.

4. Development of a formal method that can transform a production schedule into a discrete event supervisor in real time, which can realize the correct, optimized, and reactive behavior of the system determined by the scheduler.

5. Validation of the proposed hierarchical control approach by implementation in a real manufacturing setting. 


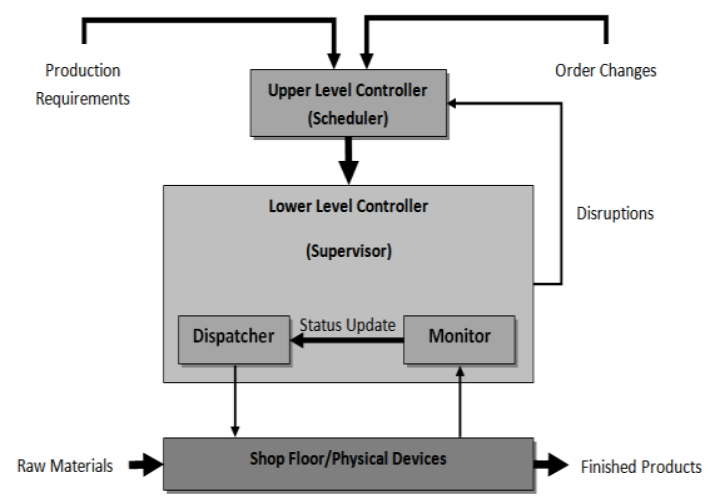

IV. Research methodology

As stated in previous chapters, myopic dispatching rules decompose the part/machine (or operation/tool) assignment into smaller sub-problems, namely machine routing (or tool selection) and part dispatching (or operation tool request prioritizing). Resources and tasks are assigned sequentially while the interaction between sub-problems due to jobs (operations) and machines (tools) becoming available at different time periods are not considered. Furthermore, the dynamic scheduling needs to be computationally efficient and to be able to handle system uncertainties such as urgent job arrivals and machine breakdowns(Bock, Stefan.,2008).

Most previous research in the dynamic scheduling of tools assumes that all operations of a part can be completed in one machine. However, in most machining systems, one part usually requires multiple palletfixture combinations to complete all operations in the same machine or in different machines. So, multiple setups for machining of a part are not uncommon in discrete part manufacturing industries.

In this research, an integrated approach to make coupled decisions about part/machine scheduling and operation/tool assignments on a rolling window basis is proposed. Specifically, a five-fold framework is proposed in this research (1) allows two levels (cell level and machine level) of hierarchical, dynamic decision making for resource and task assignment; (2) formulates the assignment decision at each level as a minimumcost flow (MCF) problem during each short-term window, and solves it by an efficient network optimization algorithm; (3) takes both the criticalities of jobs and machine reliabilities into account in decision making; (4) implements the decision for first period of each window, and reschedule resources and tasks for the remaining periods with a short-term look ahead; and (5) handles uncertainty of machine failure down time by estimating the operation completion time based on the transient analysis of machine availability for a two-state Markov process.

\section{Findings}

This research proposes a framework of a shop floor control system, which consists of a two level hierarchical scheduler for the dynamic decision of job dispatching/next machine selection and tooling schedules and a machine control scheme for operational control of jobs and tools. Most of the pre-release function is not performed before the start of a production period, so that machines, parts and tools are not assigned to each other at the planning stage. The dynamic operational decisions with minimal commands from the higher level allow the system to respond quickly to disturbances such as machine failures or demand changes. Data required for MCF model are as follows:

tw the current system time

tdi the due time of part $\mathrm{i}$

trpi the remaining processing time of part $\mathrm{i}$

tpi the processing time of task $\mathrm{i}$

tri the ready time at which the last task of part $i$ is completed

tmj the available time at which the last scheduled task on resource $\mathrm{j}$ is completed

mij the expected travel time delay of part (or tool) $i$ from its current location to machine $j$

tsi the estimated start time of task $\mathrm{i}$

tfi the estimated finish time of task i

The start time of task $\mathrm{i}$ is determined by the ready time of the task, available time of the resource, and the expected travel time delay. The expected travel time delay is the time to transfer a job from its previous operation station to its subsequent operation station at the cell-level job scheduling. At the machine level, it stands for the expected time to transport the required tool from its previous magazine to the station where the next job requests it. When tri is less than tw, it means job $\mathrm{i}$ is ready before the current time. If the time job $\mathrm{i}$ 
becomes ready plus the expected travel time delay mij is greater than the available time of machine tmj, then the machine $\mathrm{j}$ will be idle waiting for the job. Otherwise, job $\mathrm{i}$ will arrive at machine $\mathrm{j}$ early and wait for machine $\mathrm{j}$ to become available. Therefore, the task start time can be estimated by the following expression(Hany ,M.,et al.,1989):

tsi $=\max \{(\max \{$ tri, $\mathrm{tw}\}+\operatorname{mij}), \mathrm{tmj}\}$

Due to the dynamic nature of shop floor and random disruptions like machine failures, the estimates of the task ready times and the resource available times need to be updated frequently. The finish time of a task will be the start time plus the expected processing time of the task, i.e.

$\mathrm{tfi}=\mathrm{tsi}+\mathrm{E}(\mathrm{tpi})$

Since the machines are subject to random breakdowns, the processing time of the task can be estimated based on the analysis of a machine's availability. Based on the abovementioned discussion, Table 1 outlines the findings of this research in a categorized order(E,1Maraghy, H.,et al.,2009).

Table 1: Application areas of FMS in customized production.

\begin{tabular}{|c|c|c|c|c|c|}
\hline \multicolumn{4}{|c|}{ Application case(s) based on each customization method } & \multirow{2}{*}{ Type of system } & \multirow{2}{*}{ Target FMS } \\
\hline $\begin{array}{l}\text { Collaborative } \\
\text { customization }\end{array}$ & $\begin{array}{l}\text { Adaptive } \\
\text { customization }\end{array}$ & $\begin{array}{l}\text { Transparent } \\
\text { customization }\end{array}$ & $\begin{array}{l}\text { Cosmetic } \\
\text { customization }\end{array}$ & & \\
\hline$x$ & & $x$ & & \multirow{7}{*}{$\begin{array}{l}\text { Machine- } \\
\text { flexible } \\
\text { manufacturing } \\
\text { system }\end{array}$} & $\begin{array}{lll}\text { John B. Jensen, Manoj } & \text { K. } \\
\text { Malhotra, } & \text { Patrick } & \text { R. } \\
\text { Philipoom, 1996 } & \text {. }\end{array}$ \\
\hline & $x$ & & & & $\begin{array}{l}\text { Mitchell M. Tseng, Ming Lei, } \\
\text { Chuanjun Su, M. Eugene } \\
\text { Merchant, } 1997\end{array}$ \\
\hline & & $x$ & & & Geoff Buxey, 1992 \\
\hline \multirow[t]{3}{*}{$\times$} & & & & & $\begin{array}{l}\text { Robert B. Handfield, Mark D. } \\
\text { Pagell, } 1995\end{array}$ \\
\hline & & $x$ & $x$ & & F.J.A.M. van Houten, 1992 \\
\hline & $x$ & & $x$ & & $\begin{array}{l}\text { Hyung Jun Ahn, Habin Lee, } \\
\text { Sung Joo Park, } 2003\end{array}$ \\
\hline$x$ & & $x$ & & & $\begin{array}{l}\text { Mario G.C.A. Cimino, } \\
\text { Francesco Marcelloni, } 2011\end{array}$ \\
\hline & $x$ & $x$ & & \multirow{3}{*}{$\begin{array}{l}\text { Material } \\
\text { handling- } \\
\text { flexible } \\
\text { manufacturing } \\
\text { system }\end{array}$} & Qi Hao, Weiming Shen, 2008 \\
\hline$\times$ & & & & & $\begin{array}{l}\text { Jianxin Jiao, Mitchell M. } \\
\text { Tseng, 2004 }\end{array}$ \\
\hline$x$ & & & $x$ & & $\begin{array}{l}\text { James B. Dai, Neville K.S. Lee, } \\
2011\end{array}$ \\
\hline & $x$ & & & \multirow{5}{*}{$\begin{array}{l}\text { Operation- } \\
\text { flexible } \\
\text { manufacturing } \\
\text { system }\end{array}$} & $\begin{array}{l}\text { M.H.M.A. Jahromi, } \\
\text { Tavakkoli-Moghaddam,2011 }\end{array}$ \\
\hline & & $x$ & $x$ & & $\begin{array}{l}\text { Yash P. Gupta, Mahesh C. } \\
\text { Gupta, 1991 }\end{array}$ \\
\hline \multirow[t]{3}{*}{$\times$} & $x$ & & $x$ & & $\begin{array}{l}\text { Insup Um, Hyeonjae Cheon, } \\
\text { Hongchul Lee, } 2009\end{array}$ \\
\hline & $x$ & $x$ & & & $\begin{array}{l}\text { N. Suresh Kumar, R. Sridharan, } \\
2009\end{array}$ \\
\hline & $x$ & & $x$ & & 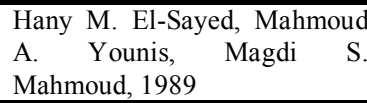 \\
\hline \multirow[t]{3}{*}{$x$} & & & & \multirow{4}{*}{$\begin{array}{l}\text { Process-flexible } \\
\text { manufacturing } \\
\text { system }\end{array}$} & Stefan Bock, 2008 \\
\hline & & $x$ & & & $\begin{array}{l}\text { H. ElMaraghy, A. Azab, G. } \\
\text { Schuh, C. Pulz, } 2009\end{array}$ \\
\hline & $x$ & $x$ & & & $\begin{array}{l}\text { Mitchell M. Tseng, Ming Lei, } \\
\text { Chuanjun Su, M. Eugene } \\
\text { Merchant, } 1997\end{array}$ \\
\hline$x$ & & & $x$ & & $\begin{array}{l}\text { Yumin He, Milton L. Smith, } \\
\text { Richard A. Dudek, } 2010\end{array}$ \\
\hline$x$ & $x$ & & & $\begin{array}{l}\text { Product- } \\
\text { flexible }\end{array}$ & $\begin{array}{l}\text { Mitchell M. Tseng, Ming Lei, } \\
\text { Chuanjun Su, M. Eugene }\end{array}$ \\
\hline
\end{tabular}




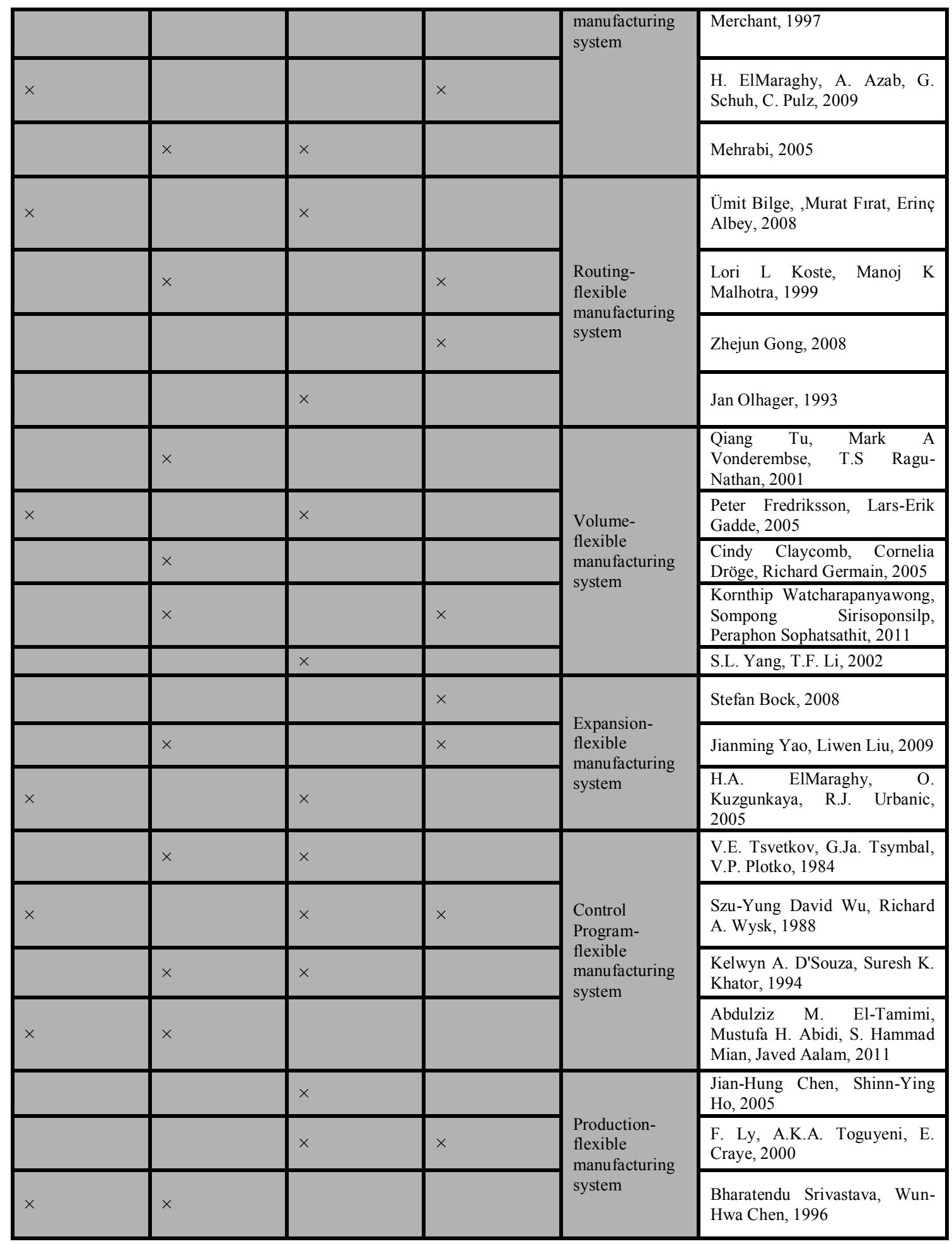

\section{Conclusion}

The contributions and conclusion of this research are summarized as follows:

(1) The proposed framework allows two levels of hierarchical, dynamic decision making for resource and task assignment. The job-machine scheduling decision is made at the cell level, while tools are scheduled for each operation sequence at the machine level. Most of the prerelease planning function is not performed, thereby allowing the system to respond quickly to disturbances such as machine breakdowns. This provides industrial software designers a better system design models for the implementation of real-time control software in complex, dynamic manufacturing systems. 
(2) The assignment decision at each level is formulated as a minimum-cost flow problem during each short-term window, and solved by the efficient network optimization algorithm. The cost coefficients corresponding to alternative assignment of resources to tasks are defined to optimize weighted completion time during each shortterm scheduling window(Yumin,He.,et al.,2010). This improves the overall due-date related system performance significantly. The sub-problem heuristics can provide researchers a good alternative algorithm to solve dynamic job scheduling problems using a rolling time horizon approach.

(3) The proposed dynamic scheduling approach is tested on multiple manufacturing system models considering variations in generic shop flow structures and configurations. The results show that the proposed approach performs significantly better than all other dispatching heuristics on Average Flow Time. It is also noticed that the performance differences between the proposed scheduling approach and the other heuristics tend to become more significant when there are more machines. It is also noticed that more operation steps, the better the proposed method performs relative to the other heuristics. The system implementers can use this result as a general design guideline to determine when the proposed approach will perform the best.

(4) The performance of the proposed approach is validated by testing a model based on a real industrial flexible manufacturing system. The study results show that the proposed scheduling approach significantly outperforms other dispatching heuristics at various levels of shop load conditions and machine breakdowns. The proposed approach is especially robust when the shop-load level increases and when there are more disruptions such as machine breakdowns.

(5) Through the investigation of the horizon length effect, it is found that the more candidate jobs in subproblems, the longer the average flow time becomes. The proposed approach performs relatively better when the average number of candidate jobs is approximately 1.5 in the cell level sub-problems and the average number of candidate operations sequences is around 2 in the machine level sub-problems. This provides system designers empirical guidelines in choosing the appropriate horizon length for implementing the proposed scheduling approach(Mehrabi,M.,2005).

(6) Research to date in scheduling with shared tools often places emphasis on the real-time control and manipulation of tools, but pay less attention to machine loading and initial tool allocations at the planning stage.

In this research, a machine-loading model is proposed to assign part types and tools to machines while considering minimum ratio of resident tools. It provides a closer coordination of the tool flow control between the tool allocation at the machine loading stage and the real-time assignment of tools at the scheduling stage.

(7) The proposed loading heuristic is tested in single stage systems. The results show that the proposed loading method performs significantly better on average flow time and tardiness than that of the random loading in most cases. It proved the research hypothesis that better system performances can be achieved by considering the ratio of resident tools in assigning part types and allocating tools to machines at the loading stage. This provides production planning practitioners a new machine loading approach producing better overall system performances when the tools are dynamically shared among machines.

VII. Future work

The presented approaches for the dynamic scheduling and machine loading with shared tools are expected to open many new topics for future research. The followings are suggested areas for the future research.

1) The automated manufacturing system models studied in this research are characterized with alternative partroutings and shared cutting tool resources. There are many other production systems that also have these characteristics. For example, in the lithography operation of wafer fabrications, the reticles are often shared among different steppers. Applying the proposed approach to the dynamic scheduling problems in these types systems and comparing its performance to those of other dispatching heuristics will be one of the areas for the future research.

2) In the simulation experiments of this research, it shows that the proposed scheduling approach performs well under some flexible flow shops and job shops when tooling resources are shared between machines. In the future research, the proposed scheduling approach should be compared to other dispatching heuristics in the generalized job shop and flow shop environments where the tools are not shared among machines.

3) In the simulation experiments of this research, exponential distribution is assumed for the machine breakdown and the repair time. In future research, it would be interesting to investigate how the proposed approach performs when the machine breakdowns follow other distributions such as Weibull and Lognormal.

4) In this research, it is assumed that the tool handling system transports tools one at a time. When a machine failed, the entire set of tools on it can be migrated to other machines in a bulk exchange manner. The proposed loading heuristic can be used to re-allocated tools and re-assign part types to other active machines, which is expected to further improve the overall system performance. Simulation experiments should be conducted to show the effectiveness of the proposed loading methods together with tool migrations in the event of machine breakdowns.

5) It is shown that better system performances can be obtained by taking into account the resident tool ratio at the loading stage in this research. It would be also interesting to investigate the performance of the proposed 
approach when the resident tool ratio is also considered in the cell-level sub-problem heuristic at the scheduling stage in future research.

\section{Acknowledgements}

The authors gratefully acknowledged to Islamic Azad University Shiraz Branch, Fars, Iran for supporting the research fund under Grant.

\section{Reference}

[1]. Abdulziz, M.,et al., 2011. "Analysis of performance measures of flexible manufacturing system, ” Journal of King Saud University - Engineering Sciences.

[2]. De Toni and S. Tonchia., 1998. "Manufacturing Flexibility: a literature review," International Journal of Production Research, vol. 36 , no. $6,1587-617$.

[3]. ElMaraghy,M., 2009. "Managing variations in products, processes and manufacturing systems, " CIRP Annals - Manufacturing Technology PP: 441-446

[4]. Hany ,M.,et al., 1989. "Modelling and simulation of a flexible manufacturing system with variable production ratios, " Applied Mathematical Modelling PP: $397-401$

[5]. Insup, Um., 2009. "The simulation design and analysis of a Flexible Manufacturing System with Automated Guided Vehicle System, ” Journal of Manufacturing Systems PP: 115-122

[6]. Jan, Olhager., 1993. "Manufacturing flexibility and profitability, ” International Journal of Production Economics PP: 67-78.

[7]. Jian,Hung Chen.,et al., 2005. "A novel approach to production planning of flexible manufacturing systems using an efficient multiobjective genetic algorithm, " International Journal of Machine Tools and Manufacture, PP: 949-957

[8]. Jianming Yao., 2009. "Optimization analysis of supply chain scheduling in mass customization, "International Journal of Production Economics PP 197-211

[9]. Kelwyn, A.,et al., 1994. "A survey of Petri net applications in modeling controls for automated manufacturing systems, " Computers in Industry PP: $5-16$

[10]. Lori L ,Koste., 1999. "A theoretical framework for analyzing the dimensions of manufacturingflexibility, "Journal of Operations Management PP: 75-93

[11]. Mehrabi,M., 2005. "Lab system design in support of manufacturing engineering curricula, " Journal of Manufacturing Systems PP: 251-255

[12]. M, Jahromi., 2011. "A novel 0-1 linear integer programming model for dynamic machine-tool selection and operationallocation in a flexible manufacturing system, "Journal of Manufacturing Systems .

[13]. Mitchell ,M.,et al., 1997. "A Collaborative Control System for Mass Customization Manufacturing, " CIRP Annals - Manufacturing Technology PP: 373-376

[14]. Nekooei,Mohammad Javad.,et al., 2012 "Designing Fuzzy Backstepping Adaptive Based Fuzzy Estimator Variable Structure Control: Applied to Internal Combustion Engine." Australian journal of basic and applied science PP: 382-398

[15]. Poorian Mahmood, Mohammad Javad Nekooei and Yusof Bin Boon. "Academic Cheating In Higher Educationthe Effect Of A Student Development Approach A Study at Universiti Teknologi Malaysia." IOSR Journal of Research \& Method in Education (IOSR-JRME),Vol.1, pp.40-3.http://www.iosrjournals.org/iosrjrme/ pages/v1-i6.html.

[16]. Qiang,Tu.,et al., 2001. “The impact of time-based manufacturing practices on mass customization and value to customer, ” Journal of Operations Management PP: 201-217

[17]. Stefan ,Bock., 2008. "Supporting offshoring and nearshoring decisions for masscustomization manufacturing processes, "European Journal of Operational Research PP: 490-508

[18]. Szu,Yung David Wu.,et al., 1988. "Multi-pass expert control system - a control/scheduling structure for flexible manufacturing cells, ” Journal of Manufacturing Systems PP: 107-120

[19]. V.E, Tsvetkov., 1984. "Computer-aided design of production processes and control programs for flexible manufacturing systems," Computers in Industry PP: $261-263$

[20]. V, Malhotra., et al., 2010. "flexible manufacturing systems, " International Journal of Engineering Science and Technology PP: 137-142

[21]. W.E, Wilhelm., 1986. "The application of lognormal models of transient operations in the flexible manufacturing environment, " Journal of Manufacturing Systems PP: 253-266

[22]. Yash P, Gupta., et al., 1991. "Flexibility and availability of flexible manufacturing systems: An information theory approach, "Computers in Industry PP: 391-406

[23]. Yumin, He., 2010. "Robotic material handler scheduling in flexible manufacturing systems for mass customization, " Robotics and Computer-Integrated Manufacturing PP: 671-676

[24]. Zhejun, Gong., 2008. "An economic evaluation model of supply chain flexibility, " European Journal of Operational Research PP: 745-758 